\title{
ENERGY SAVINGS IN APPLICATIONS FOR WIRELESS SENSOR NETWORKS TIME CRITICAL REQUIREMENTS
}

\author{
Tran Cong Hung ${ }^{1}$ and Ho Huu Trung ${ }^{1,2}$ \\ ${ }^{1}$ Post \& Telecommunications Institute of Technology, Vietnam \\ ${ }^{2}$ Ho Chi Minh City University of Technology, Vietnam
}

\begin{abstract}
Along with handling and poor storage capacity, each sensor in wireless sensor network (WSN) is equipped with a limited power source and very difficult to be replaced in most application environments. Improving the energy savings in applications for wireless sensor networks is necessary. In this paper, we mainly focus on energy consumption savings in applications for wireless sensor networks time critical requirements. Our Paper accompanying analysis of advanced technologies for energy saving techniques for the optimization of energy efficiency together with the data transmission is optimal. Moreover, we propose improvements to increase energy savings in applications for wireless sensor networks require time critical (LEACH improvements). Simulation results show that our proposed protocol significantly better than LEACH about the formation of clusters in each round, the average power, the number of nodes alive and average total received data in base stations.
\end{abstract}

\section{KEYWORDS}

Sensor network, energy efficiency, routing

\section{INTRODUCTION}

Wireless sensor networks (WSN) [1] became famous with the advancement of technology MEMS (Micro Electro Mechanical Systems) the sensors with battery power source support very small devices thus limiting energy consumption leading to energy and data transmission over a period allowing an important issue in WSN. All sensors feel environmental and transmit data to the Base Station (BS), each cluster has a Cluster Head $(\mathrm{CH})$ will communicate with all members of the cluster. $\mathrm{CH}$ will transmit all data to the BS. In the clustering technique can reduce the cost of communication of nodes because the nodes only need to send data to $\mathrm{CH}$ nearest. However $\mathrm{CH}$ will use more energy than normal nodes to communicate with the BS. Experts have been studying improvements in optimization algorithms to optimize performance to improve the efficiency of use of sensor networks.

Two important issues are addressed is fundamental to minimize energy consumption and reduce time delays in data transmission to optimize network longevity. Clustering techniques [2] has emerged as a popular option to achieve energy efficiency and scalability in large-scale sensor networks [3-5]. Using Clustering, sensors can be managed locally by a cluster, a $\mathrm{CH}$ to manage the cluster and is responsible for communications between the cluster and the base station. Clustering provides a framework to facilitate the management of resources. Clustering can support many important features in a cluster of devices, such as channel access for members in cluster and control, routing and code splitting to avoid noise inter-cluster. Moreover, it helps distributed management responsibility from the base station to cluster head of devices [6]... Some sensors can be moved, by attaching sensors to the mobile device, as being achieved in the project Robomote [7].

DOI: $10.5121 / \mathrm{ijcnc} .2016 .8403$ 
In recent years, there are a lot of routing protocols in WSN are used that a few of them have considered the requirements of network applications in time critical data transmission is important [8-15]. In wireless sensor network gathers data from the relevant data would reduce the large amounts of data traffic on the network, avoid information overload, creating a more accurate signal and requires less energy than sending all unprocessed data inside the network. In this paper, we propose a protocol developed from LEACH to save energy in real-time applications in wireless sensor networks to maximize the lifetime of the network, reducing energy consumption and time critical data transmission. LEACH improvements considered higher priority for time critical data than the data space of time vital so that critical data is immediately transmitted to the base station. LEACH improved using cluster head selecting different for normal nodes and advance nodes, besides using the threshold values to reduce data transmission delay time is important. LEACH improvement organized sensor nodes into the cluster, each node in the cluster sensor signal received from a neighbor node before and passed to a next door neighbor node. LEACH improvement uses a threshold value for reducing transmission delay of time critical data. LEACH also improved through a transmission mechanism cluster-chain increases for sending data packets from cluster head to the base station. Through the simulations compared with previous LEACH, show that the proposals protocol we can do better in increasing the lifespan of the network, the number of sensor node alive, time data transmission stability, low power consumption, total data received in the base station higher. In this paper we present the first part is the introduction, part 2 is the related work, part 3 presents the network model, part 4 mentioned the Proposed protocol, part 5 evaluation and implementation and part 6 conclusion.

\section{Related Work}

Recently, there are many different clustering techniques to reduce energy consumption capacity of sensor nodes were developed. Group a large number of sensor nodes into the cluster and keep them constant communication is quite complicated. Here, we mention some of the most recent works with different perspectives in order to increase its ability to improve the energy-saving properties of the nodes.

LEACH [8] protocol hierarchical cluster low-energy adaptation. As one of the routing protocols are the most popular clustering in wireless sensor networks. LEACH is activities are divided into rounds, where each round starts with a stage setup for the formation of clusters, followed by a steady state phase, where each round begins with a period set to the formation of clusters. Leach will randomly select a few nodes is clusters head $(\mathrm{CH})$ and will rotate roles to load balance the energy of sensor nodes in the network. In a cluster all node is not $\mathrm{CH}$ will transmit its data to the $\mathrm{CH}$. Cluster head nodes receive data from all nodes in the cluster membership, implementing local data processing to reduce the amount of data and transmission of duplicate data, and then transmitted to the base station. Therefore, becoming $\mathrm{CH}$ will consumable more energy than the node is not selected do $\mathrm{CH}$. Data collection is performed periodically. LEACH protocol provides Energy Efficiency but the expected number of clusters is predefined. Another disadvantage is that it does not guarantee $\mathrm{LEACH}$ distributed $\mathrm{CH}$ good and energy consumption assumed not uniform for the $\mathrm{CH}$.

PEGASIS [9] is an improvement of the well-known protocol LEACH. PEGASIS applied in the network model including a set of nodes that are uniformly distributed over a geographic area. In which each nodes knows the location information of other nodes in the network. Besides we also have the ability to capacity control and covered an arbitrary area. Responsibilities of this nodes leaves collecting and transmitting data to the base station. The aim is to develop a routing architecture and a centralized data scheme to minimize power consumption and transmits data to the base station centralized with the minimum latency while still balancing capacity of consumption among nodes in the network. 
Using PEGASIS will solve overhead problems caused by the formation of clusters in LEACH, reduce the number of transmitting and receiving data by the set of data. However PEGASIS have large transmission latency for remote nodes in the chain. Furthermore, bottleneck effect can occur in the $\mathrm{CH}$ node.

TEEN [10] is the energy efficient protocol sensing threshold level given for the time-dependent applications. In this protocol sensor nodes continuously sense the environment, but do not regularly send data (the process of sending data are controlled by threshold values). $\mathrm{CH}$ sent to component clusters a hard threshold value $(\mathrm{Ht})$, which the threshold value of the attribute is felt and a soft threshold value (St) which is a minor change in the value of the attribute making nodes switch to sending data mode. The hard threshold value is to reduce the transmission by only allowing transmission node when attribute feel in a proper range. Soft threshold value is to reduce further transmission times when there is little change of attributes that need to be measured (when the change is smaller than the soft threshold, no data transmission). The smaller the soft threshold value, the higher accuracy of the network, but also increase energy costs. Hence it should have the balance between the accuracy and power consumption. When $\mathrm{CH}$ nodes change, the threshold values will change and advertise. The main disadvantage of this protocol is if the node not receive the threshold values of the cluster head sent to the node,the node will not send data, therefore $\mathrm{CH}$ will not receive data over the entire network. In addition, it also assign time slot when all the nodes are enabled transmitting and sending data simultaneously, it cannot distinguish among failed nodes or big changes in attribute values.

ECCPTC [11] is recommended to save energy consumption in applications for wireless sensor networks requiring time critical in terms of network cluster-chain, to maximize the network life, minimize energy consumption and reduce the time critical data transmission. ECCPTC considered higher priority for time critical data than the data space of time vital so that critical data is immediately transmitted to the base station. ECCPTC using a threshold value to reduce the delay time of transferring important data. ECCPTC organized sensor nodes into the cluster and form a chain between sensor nodes, each sensor node in the cluster received from a previous neighbor node and passes to a next neighbor node. Cluster Head was elected based remaining energy of the nodes, the distance from the neighboring node and the neighbor nodes of the node. ECCPTC also undergoes a transmission chain mechanism for sending data packets from the host device to the base station cluster.

\section{NETWORK MODEL}

In this paper, we considered a sensor network including $\mathbf{n}$ sensor nodes be unified the following attributes.

- Each node performs sensor tasks periodically and always have the data to send to the base station.

- A fixed base station can be placed inside or outside the sensor network.

- All nodes are standing still and is fixed energy.

- The control nodes have the ability to control the energy resources of its energy transmission.

- All nodes have the ability to operate in cluster head mode and send data mode.

- The synthesis and handling of data has been used before to reduce the transmission of all data. 
The energy patterns as in LEACH was used to calculate the energy transmission, $E_{T x}$ symbol is used in the transmission of a k-bit message in the distance $d$, the energy consumed by the waves propagate be given by the following formula:

$E_{T x}(k, d)=\left\{\begin{array}{l}k E_{\text {elec }}+k E_{f s} d^{2}, d<d o \\ k E_{\text {elec }}+k E_{m p} d^{4}, d \geq d o\end{array}\right.$

Where:

$$
\begin{aligned}
& \mathrm{k}: \text { number of bits transmitted } \\
& \mathrm{E}_{\mathrm{elec}} \text { : The energy consumed to transmit or receive data. } \\
& \varepsilon_{\mathrm{mp}}: \text { Amplification coefficient of energy transfer. } \\
& \varepsilon_{\mathrm{fs}}: \text { Energy loss in space } \\
& d o: \text { Distance from the BS to the receiving node, } \varepsilon_{\mathrm{mp}} \text { or } \varepsilon_{\mathrm{fs}} \text { depend on } d o \text {. } \\
& d o=\sqrt{\frac{\varepsilon_{f s}}{\varepsilon_{m p}}}
\end{aligned}
$$

To receive k bits of data are $E_{R x}$ energy consumption:

$$
E_{R x}=k * E_{\text {elec }}
$$

Electronic energy $\left(\mathrm{E}_{\text {elec }}\right)$, depends on factors such as digital coding, modulation, filtering and the spread of the signal, while the energy amplifier, $E_{f s} d^{2}$ or $E_{m p} d^{4}$ depends on the distance to the reception apparatus and bit error rate acceptable.

From equation (3), we can see that the process of receiving data is a procedure also consume high cost in transmission data. Thus, the number of active transmission and reception must be cut in order to reduce energy consumption. If a node using $E_{D A}$ synthesis energy to a little data, then energy is used to aggregate the data packet $m$ into a single package is Eq.

$E_{q}(m, k)=m * k * E_{D A}$

In LEACH, delay for the transmission of data packets to the head of the group depending on the maximum number of nodes in the cluster. Among all similar sized clusters and K clusters in the network, a maximum delay of one round is calculated as the equation.

$T=\left(\frac{N}{K}-1\right)+K+C$

\section{Proposed Protocol}

In the process of understanding LEACH algorithm and other algorithms we noticed that, extent wants to become node $\mathrm{CH}$ in a round must be reconsidered in normal circumstances and in special cases (energy level of node after several rounds remain high).

* Choose node cluster head in LEACH. 
When the cluster is created, each node $\mathbf{n}$ automatically decide it is node $\mathrm{CH}$ to the next round or not. The selection process takes place as follows: each sensor node randomly select a number between 0 and 1 . If this number is smaller than the threshold $\mathrm{T}(\mathrm{n})$, then it becomes node $\mathrm{CH}$. T(n) is determined by the following equation:

$T_{1}(n)=\frac{P}{1-P *\left(\operatorname{rmod} \frac{1}{p}\right)}$

With these node without doing $\mathrm{CH}$ 1/P final round, by contrast $\mathrm{T}_{1}(\mathrm{n})=0$.

Where:

P: Level of desire to become cluster head $(0<\mathrm{P}<1)$.

r: Number of rounds.

Using this algorithm, each node will be the correct $\mathrm{CH}$ node once for $1 / \mathrm{P}$ round.

Note: after 1/P-1 round, $\mathrm{T}_{1}(\mathrm{n})=1$ with all the nodes that have not been do $\mathrm{CH}$.

* Here we propose extent wish to become node $\mathrm{CH}$ of one round with the two cases as follows:

Case 1: The probability $\mathrm{CH}$ elected by normal node with $\mathrm{k}$ bits of data we call that pn.

$$
p n=\frac{p}{k+d o * q * \log \left(\frac{n}{r}\right)}
$$

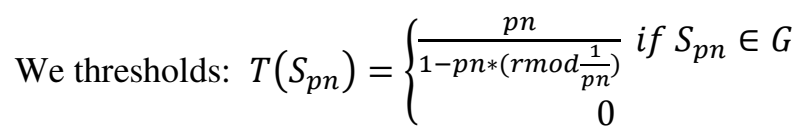

Case 2: The probability $\mathrm{CH}$ elected by advance node with $\mathrm{k}$ bits of data we call that pa.

$p a=\frac{p}{m\left(k+d o * q * \log \left(\frac{n}{r}\right)\right)}$

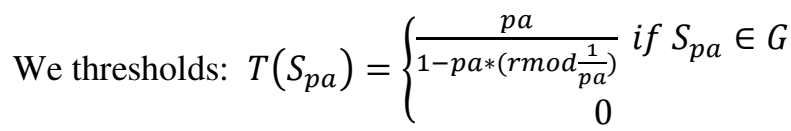

Where:

p: Level of desire to become cluster head $(0<\mathrm{p}<1)$.

do: Distance from the BS to the receiving node

q: Wrong number occurs, this value very small at 0.01

$\mathrm{m}$ : Percentage of nodes than are advanced

$\mathrm{n}$ : Number of Nodes in the field

r: Number of rounds

$\mathrm{G}$ : is the set of normal nodes or advanced nodes which can become $\mathrm{CH}$ 
Corresponding to the nodes in the network will receive different energy levels will be received probability calculations differently $\mathrm{CH}$ node, when the value energy is 0 we use pn, when the value energy is 1 we use pa to determine $\mathrm{CH}$.

In addition, to the process of forming clusters is faster and minimize data transmitted waste to BS. In this algorithm, we also set the threshold value it is devoted to attribute to feel, to datacasting better.

Hard threshold $(\mathrm{Ht})$ : The hard threshold value is to reduce the transmission by only allowing transmission node when attribute feel in a proper range, nodes induction to turn on this value transmitter and report to their $\mathrm{CH}$.

Soft threshold (St): The soft threshold value is to reduce further transmission times when there is little change of attributes that need to be measured (when the change is smaller than the soft threshold, no data transmission).

The nodes to feel continuously environment. The first time a parameter from the attribute set reaches its hard threshold value, the node switches on its transmitter and sends the sensed data. The sensed value is stored in an internal variable in the node, called the sensed value (sv). From these changes, we call our proposed protocol is a protocol based on improved algorithm LEACH.

\section{EVALUATION AND IMPLEMENTATION}

In this section, we perform assessment LEACH with LEACH improvements on MATLAB and compare its performance in the average energy consumption, Average data is sent to base station, dissipated energy, the number of alive nodes and dead nodes through the round.

\subsection{Simulation Settings}

We ran simulations on MATLAB, for the 300 nodes in the network $100 \mathrm{~m} \times 100 \mathrm{~m}$, with unequal energy between the nodes to show the uneven influence between nodes in the network. Location BS located at (150.50), length of per message is 4000 bytes, the energy lose when data transmission and energy lost when receipt the data is $\mathrm{E}_{\text {elec }}=50 \mathrm{~nJ} / \mathrm{bit}$, transmit amplifier types $\mathrm{E}_{\mathrm{fs}}=10 \mathrm{pJ} / \mathrm{bit} / \mathrm{m}^{2}$ and $\mathrm{E}_{\mathrm{mp}}=0.0013 \mathrm{pJ} / \mathrm{bit} / \mathrm{m}^{4}$, Maximum number of rounds is 100 laps. As for improved algorithms we use the hard threshold $\mathrm{h}=100$ and soft threshold $\mathrm{s}=2$, the sensed value $\mathrm{sv}=0$ to determine the time of data transmission.

Table 1: Simulation parameter

\begin{tabular}{|l|l|}
\hline Parameter & Value \\
\hline Network size & $100 \mathrm{~m}^{*} 100 \mathrm{~m}$ \\
\hline Number of nodes $(\mathrm{n})$ & 300 \\
\hline Rate to become cluster head $(\mathrm{p})$ & 0.2 \\
\hline Base station location $\left(\mathrm{X}_{\mathrm{BS}}, \mathrm{Y}_{\mathrm{BS}}\right)$ & $(150,50)$ \\
\hline Data packet size & $4000 \mathrm{bit}$ \\
\hline Initial energy $\left(\mathrm{E}_{0}\right)$ & $0.1 \mathrm{~J}$ \\
\hline $\mathrm{E}_{\text {elec }}$ & $50 \mathrm{~nJ} / \mathrm{bit}$ \\
\hline $\mathrm{E}_{\mathrm{mp}}$ & $0.0013 \mathrm{pJ} / \mathrm{bit} / \mathrm{m}^{4}$ \\
\hline $\mathrm{E}_{\mathrm{fs}}$ & $10 \mathrm{pJ} / \mathrm{bit} / \mathrm{m}^{2}$ \\
\hline & $\sqrt{\frac{E f s}{E m p}}=87 \mathrm{~m}$ \\
\hline do & $\mathrm{h}=100$ \\
\hline High threshold & $\mathrm{s}=2$ \\
\hline Soft threshold & \\
\hline
\end{tabular}




\subsection{Simulation Results}

Figure 1(a) and 1(b) presented results for the 300 node clustering randomized, as can be seen the proposed protocol has better network clustering, the amount of each cluster node spread evenly and distance from $\mathrm{CH}$ to the members of the cluster node short.

- Red node showing the energy node has been exhausted and can't exist, the node can't continue to transmit data

- Shown the node Normal

+ Shown the node Advanced

- Shown the node Normal was selected as $\mathrm{CH}$

* Shown the node Advanced was selected as $\mathrm{CH}$

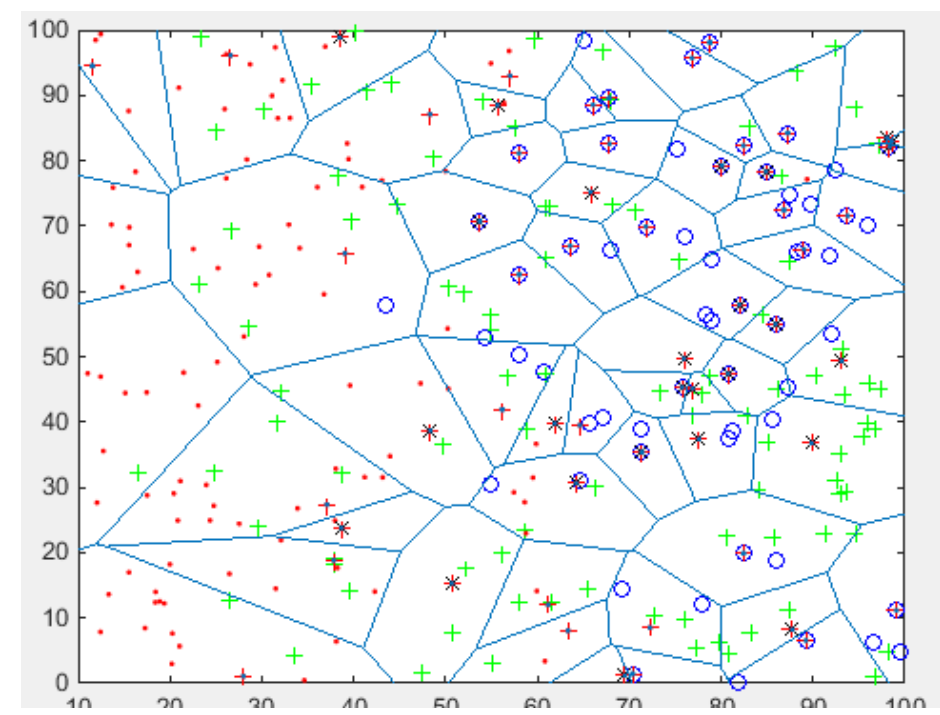

(a)

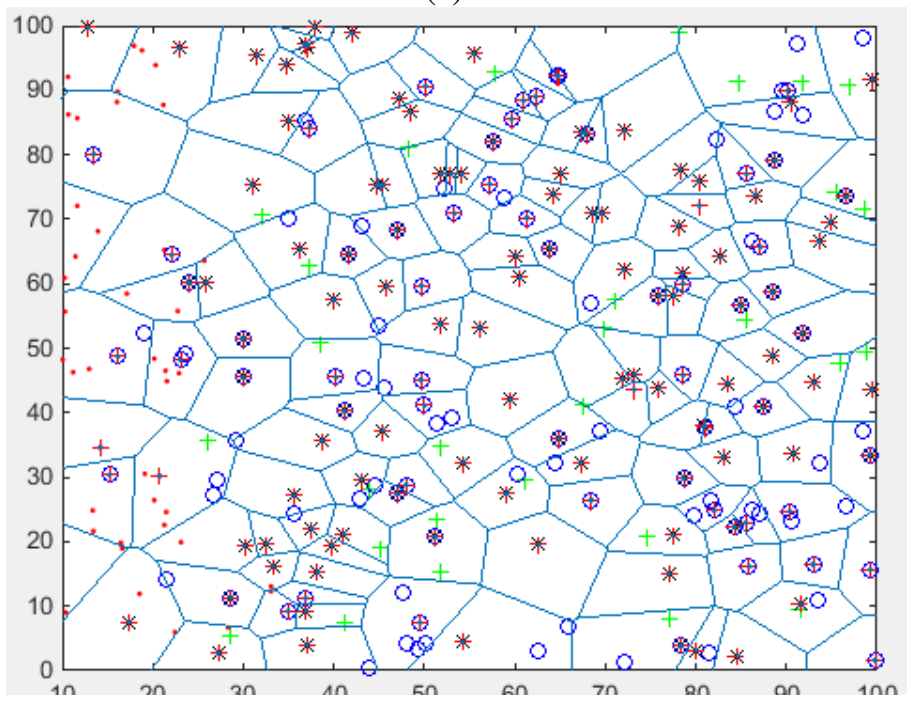

(b)

Figure 1. Results of partitioning the network (a) LEACH, (b) LEACH improvements 
Figure 2 shows the average energy of each node after each round. The average energy of the LEACH after 100 rounds of $0.04(\mathrm{~J})$ whereas Leach improvement of $0.06(\mathrm{~J})$. Thanks to optimal clustering method leads to distance between $\mathrm{CH}$ and nodes in the short clusters should the node power consumption significantly reduced.

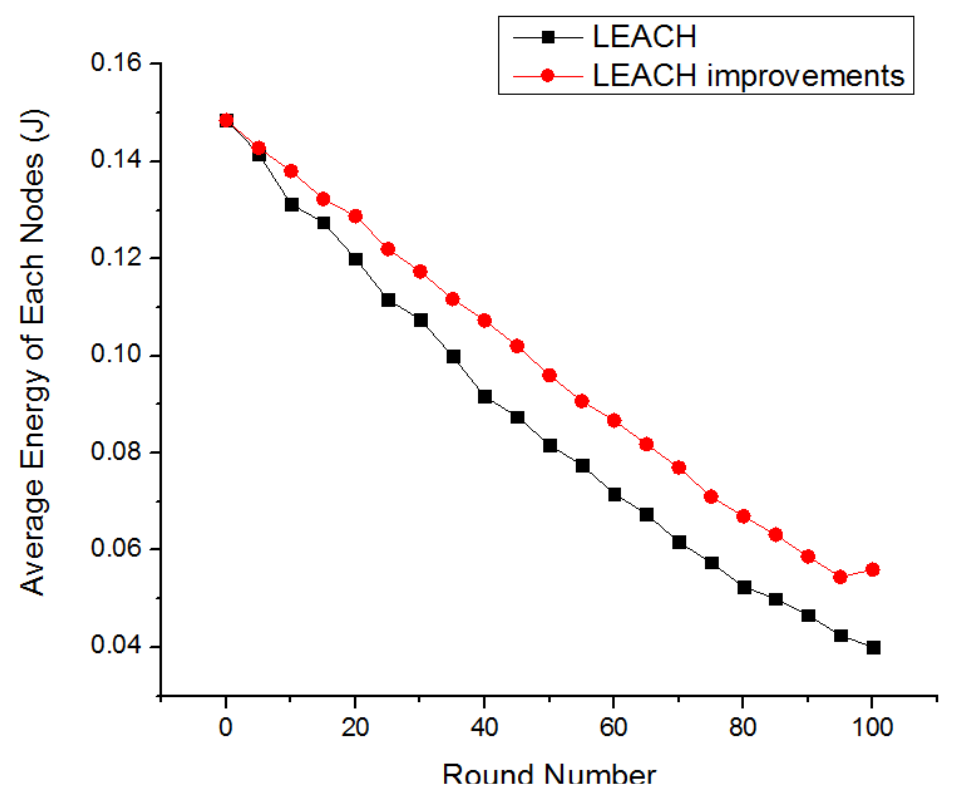

Figure 2. Average energy of each node after each round

Figure 3 shows the energy dissipated of the algorithm, is clearly improved algorithm uses less energy than LEACH. With the initialization energy is $0.1(\mathrm{~J})$ after 100 rounds, LEACH energy level of remaining $0.01(\mathrm{~J})$ while that the LEACH improvement is $0.07(\mathrm{~J})$. Reduce energy dissipation is mainly based on the selection of a $\mathrm{CH}$ selective manner and distance data transmission is minimized.

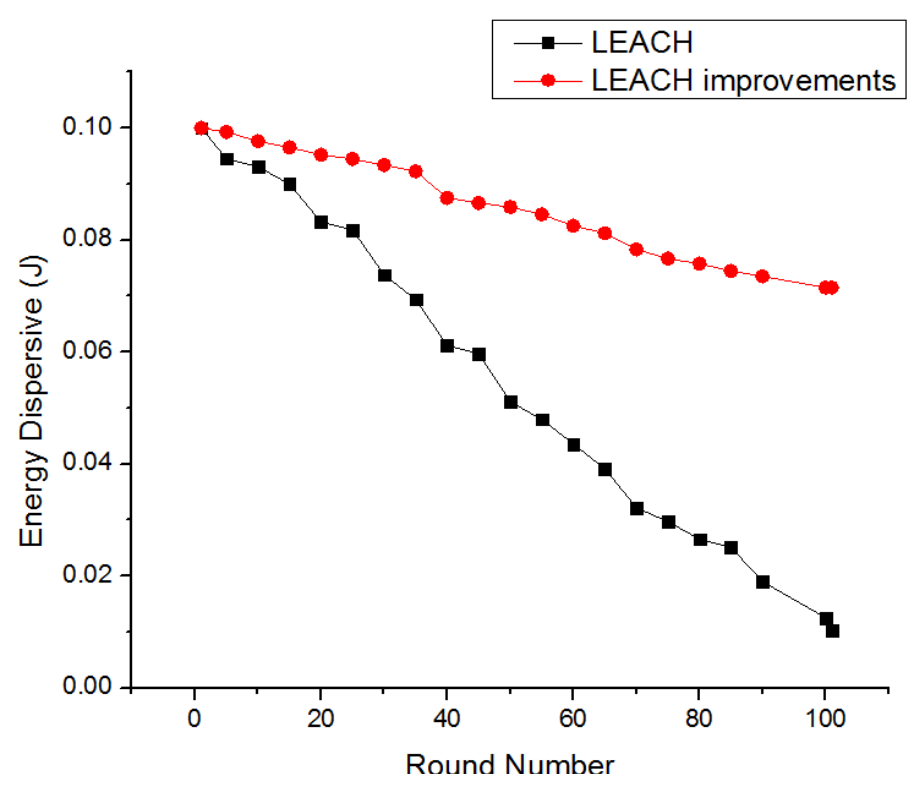

Figure 3. Energy dissipated 
Figure 4 and figure 5 and show the number of nodes dead and the number of nodes alive after each round. Here with 300 nodes initially and after 100 rounds, number of node died of the LEACH improvement is 70 nodes much lower compared LEACH is 120 nodes. Protocol our proposals could extend the life of the network than LEACH. Because our protocol selection $\mathrm{CH}$ a more selective manner and besides the data transmission of the nodes is managed by the threshold level has brought better results.

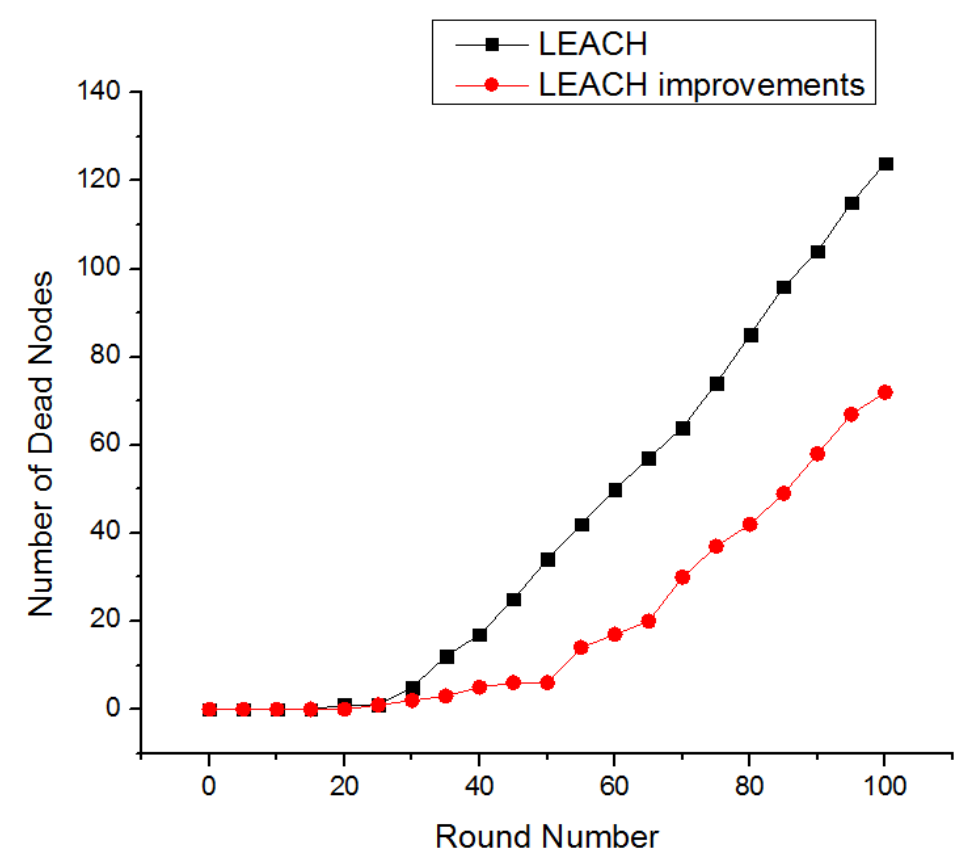

Figure 4. The number of dead node

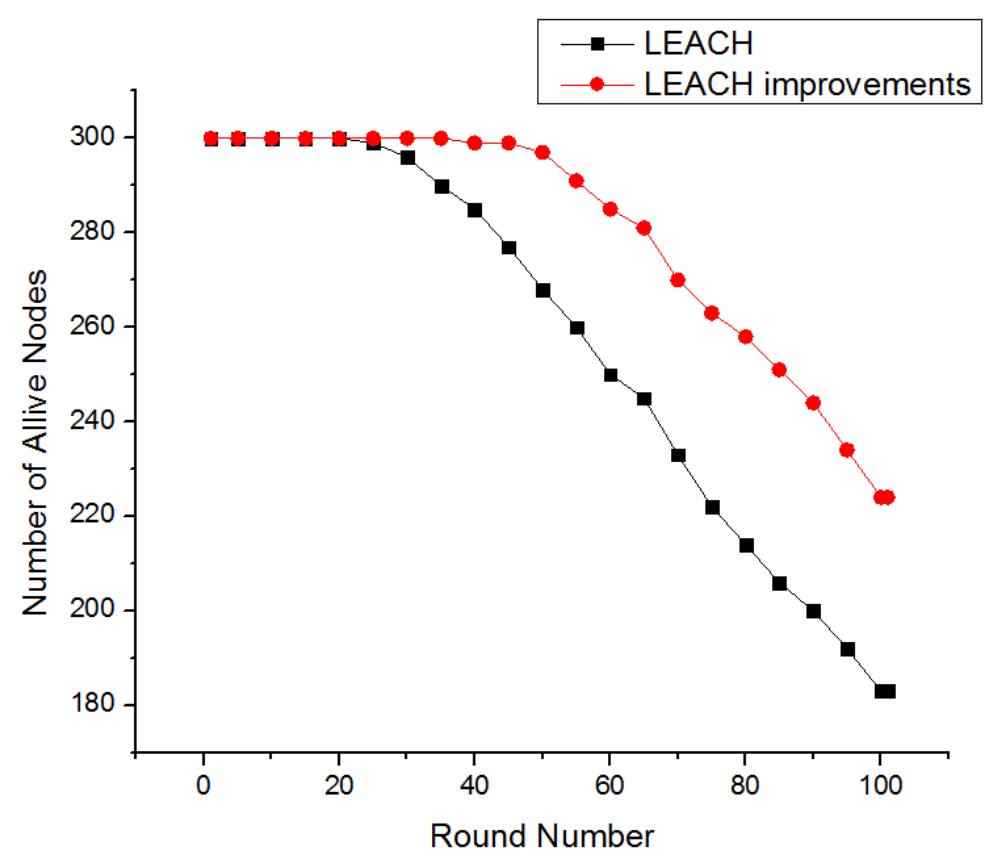

Figure 5. The number of alive node 
Figure 6 show the average number node $\mathrm{CH}$ to send the packet to the $\mathrm{BS}$, here improvements algorithm will be getting larger the number of packets transmitted from $\mathrm{CH}$ to the $\mathrm{BS}$, ensure that the data to destination time critical.

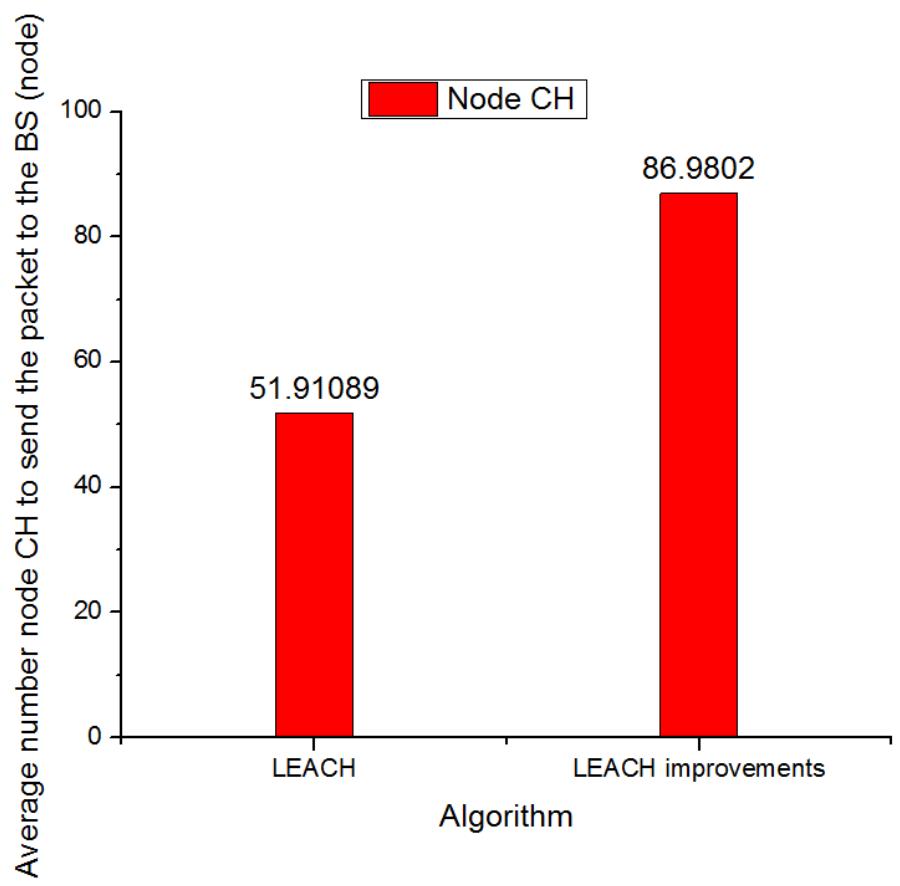

Figure 6. Average number node $\mathrm{CH}$ to send the packet to the Base Station.

Figure 7 and figure 8 shows the number of advanced nodes and normal node through the round dead, the number of node dead improvements algorithm is more optimal.

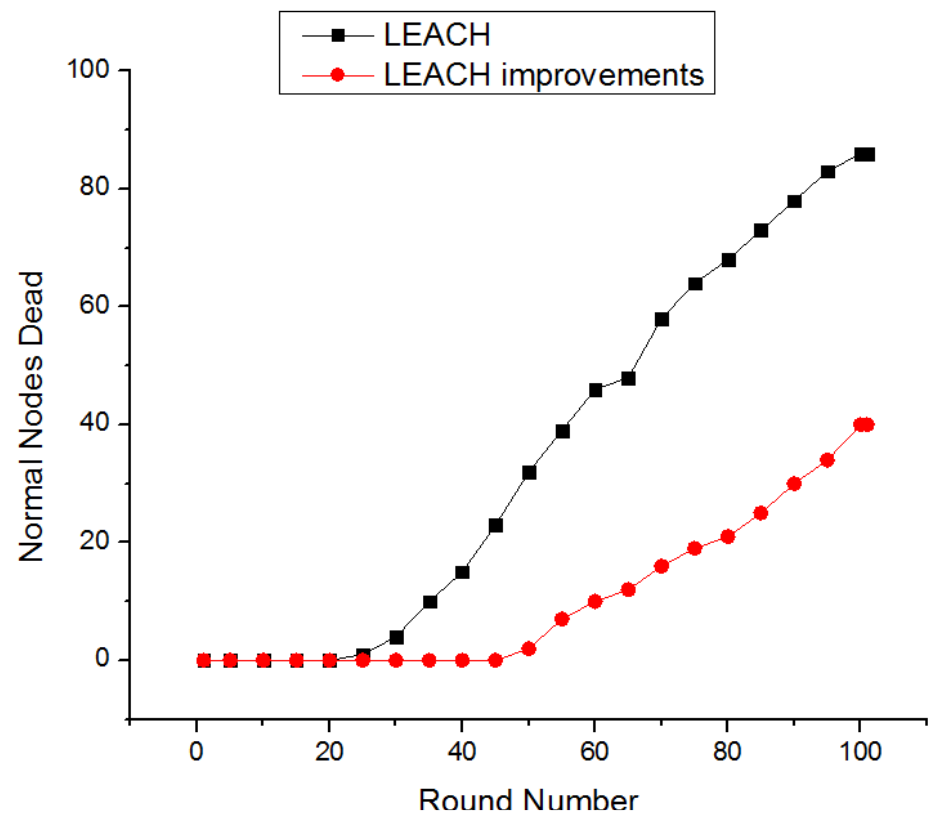

Figure 7. Number of Normal nodes dead 
International Journal of Computer Networks \& Communications (IJCNC) Vol.8, No.4, July 2016

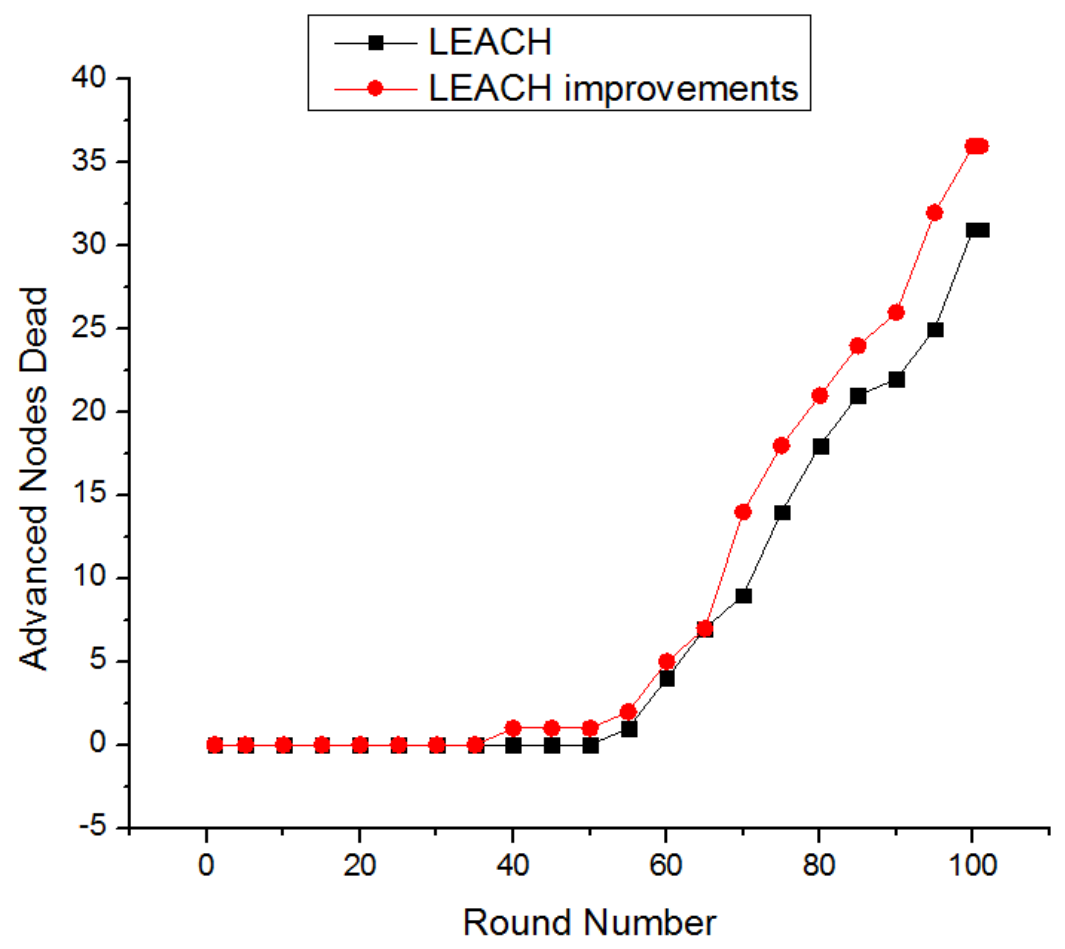

Figure 8. Number of Advanced nodes dead

\section{Conclusions}

Range of WSN applications is huge, can adapt flexibly in handling and troubleshoot upon the occurrence of damage, network deployment costs low,... However the design of a WSN works well, flexibility, easy to implement practical applications faced many difficulties because many causes, of which energy of nodes is limited and difficult to reloaded is the problem of urgency. Therefore if using energy sources available on the node will cause inefficient communication process interruption, the network becomes fragmented. In this paper we show the main characteristics of routing clustering methods to increase energy efficiency. We also have simulation algorithms to see their effect. In the future, development direction of the paper is to implement improvements increase algorithm operational efficiency of sensor networks, energy efficiency and minimizing data transmission time. Install the new embed code to the node (sensor) to put into practice.

\section{REFERENCES}

[1] Nor Azlina Ab. Aziz, Kamarulzaman Ab. Aziz (2011), "Managing disaster with wireless sensor networks", ICACT2011.

[2] Akkaya K, Younis M (2005), “A survey on routing protocols for wireless sensor networks”, Elsevier J Ad Hoc Netw 3(3):325-349.

[3] Chen G, Li Ch and Ye M (2009), "An unequal clusterbased routing protocol in wireless sensor networks", Wireless Network. 15(2), 193-207.

[4] Chang JH (2010), "An energy-aware, cluster-based routing algorithm for wireless sensor networks", J. Inform. Sci. Eng. (26), 2159-2171.

[5] Aslam N, Phillips W, Robertson W and Sivakumar Sh (2011), "A multi-criterion optimization technique for energy efficient cluster formation in wireless sensor networks", Inform Fusion. 12(3), 202-212. 
International Journal of Computer Networks \& Communications (IJCNC) Vol.8, No.4, July 2016

[6] Denga J, Hanb YS, Heinzelman WB and Varshneya PK (2011), "Balanced-energy sleep scheduling scheme for high-density cluster-based sensor networks", Elsevier J. Comput Comm, 28(14), 16311642.

[7] Dantu K, Rahimi M, Shah H, Babel S, Dhariwal A, and Sukhatme G. (2005), "Robomote: Enabling Mobility In Sensor Networks", Los Angeles, CA 90089.

[8] Heinzelman W, Chandrakasan A, Balakrishnan H (2002), "An application-specific protocol architecture for wireless micro sensor networks", IEEE, 660-670.

[9] Lindsey S, Raghavendra C (2002), "PEGASIS: power-efficient gathering in sensor information systems”, IEEE Aerosp Conf Proc 3:1125-1130.

[10] Manjeshwar A, Agarwal D.P (2001), “TEEN: a routing protocol for enhanced efficiency in wireless sensor networks”, ISSN 1530-2075, IEEE, San Francisco, CA, USA, pp 2009 - 2015.

[11] Razieh S, Sam J (2012), “An energy efficient cluster-chain based routing protocol for time critical applications in wireless sensor networks", iSee.

[12] Elham Rezaei, Amir Abbas Baradaran \& Atefeh Heydariyan (2015), "Multi-hop Routing Algorithm Using Steiner Points for Reducing Energy Consumption in Wireless Sensor Networks", Springer Science+Business Media New York.

[13] Hamid Barati, Ali Movaghar \& Amir Masoud Rahmani (2015), "EACHP: Energy Aware Clustering Hierarchy Protocol for Large Scale Wireless Sensor Networks", Springer Science+Business Media New York.

[14] A. Haider, M. Sandhu, N. Amjad, H. Ahmed, J. Ashraf, A. Ahmed, Z. A. Khan, U. Qasim, N. Javaid (2014), "REECH-ME: Regional Energy Efficient Cluster Heads based on Maximum Energy Routing Protocol with Sink Mobility in WSNs" ISSN 2090-4304, Journal of Basic and Applied Scientific Research.

[15] Abdul Razaque, Musbah Abdulgader, Chaitrali Joshi, Fathi Amsaad, Mrunal Chauhan (April-2016). "P-LEACH: Energy efficient routing protocol for Wireless Sensor Networks", IEEE.

\section{AuTHORS}

Tran Cong Hung was born in Vietnam in 1961. He received the B.E in electronic and Telecommunication engineering with first class honors from HOCHIMINH University of technology in Vietnam, 1987. He received the B.E in informatics and computer engineering from HOCHIMINH University of technology in Vietnam, 1995. He received the master of engineering degree in telecommunications engineering course from postgraduate department Hanoi University of technology in Vietnam, 1998. He received Ph.D at Hanoi University of technology in Vietnam,

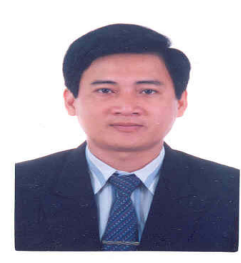
2004. His main research areas are B - ISDN performance parameters and measuring methods, QoS in high speed networks, MPLS. He is, currently, Associate Professor PhD. of Faculty of Information Technology II, Posts and Telecoms Institute of Technology in HOCHIMINH, Vietnam.

Ho Huu Trung was born in Vietnam in 1991. He received the B.E in informatics and computer engineering from HOCHIMINH City University of Technology, Vietnam, 2013. He is currently a MSc. Candidate in Information System from Post \& Telecommunications Institute of Technology, Vietnam in 2016. He is working as lecturer in Faculty of Information Technology and Vocational Training, HOCHIMINH City University of Technology, Vietnam.

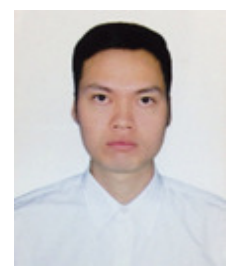

